\title{
On conformal higher spin wave operators
}

\author{
Teake Nutma and Massimo Taronna \\ Max-Planck-Institut für Gravitationsphysik, Albert Einstein Institut \\ Am Mühlenberg 1, 14476 Golm, Germany \\ E-mail: teake.nutma@aei.mpg.de, massimo.taronna@aei.mpg.de
}

ABSTRACT: We analyze free conformal higher spin actions and the corresponding wave operators in arbitrary even dimensions and backgrounds. We show that the wave operators do not factorize in general, and identify the Weyl tensor and its derivatives as the obstruction to factorization. We give a manifestly factorized form for them on (A)dS backgrounds for arbitrary spin and on Einstein backgrounds for spin 2. We are also able to fix the conformal wave operator in $d=4$ for $s=3$ up to linear order in the Riemann tensor on generic Bach-flat backgrounds.

Keywords: Higher Spin Symmetry, Gauge Symmetry, Conformal and W Symmetry, Space-Time Symmetries

ARXIV EPRINT: 1404.7452 


\section{Contents}

1 Introduction $\quad 1$

2 Conformal higher spin fields $\quad 3$

3 Factorization of conformal wave operators 4

3.1 Ansatz 4

$\begin{array}{ll}3.2 & \text { Arbitrary spins on AdS backgrounds }\end{array}$

3.3 Spin 2 on generic backgrounds 8

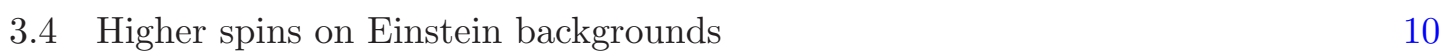

4 Spin 3 wave operator on Bach-flat backgrounds 12

5 Conclusions 13

$\begin{array}{ll}\text { A Notation and conventions } & 14\end{array}$

B Spin s wave operator in standard tensor notation $\quad 16$

$\begin{array}{ll}\text { C Wave operator in non factorized form } & 17\end{array}$

D Examples in various dimensions $\quad 19$

$\begin{array}{ll}\text { D.1 Spin } 1 \text { data } & 19\end{array}$

$\begin{array}{lll}\text { D.2 Spin } 2 \text { data } & 19\end{array}$

\section{Introduction}

Conformal gauge theories have received quite some attention over the years. In particular, the actions of Weyl gravity and conformal supergravity, together with their corresponding wave equations, have been studied in great detail [1-11] as natural extensions of ordinary gravity and supergravity theories. Interest has been also devoted to the corresponding higher spin generalizations [12-18], not just because of the intriguing role of conformal symmetry. Flat space higher spin (HS) fields are namely naturally endowed with higher derivative linearized curvatures [19] that play a key role in conformal gauge theories. ${ }^{1}$

More recently, conformal HS fields have found interesting applications in the context of the AdS/CFT correspondence. There, they play the role of sources to the conformal currents, defined in the free $\mathrm{O}(\mathrm{N})$ vector models as well as in generic CFT's in their free limit [25-32].

\footnotetext{
${ }^{1}$ See $[20-24]$ for some reviews of HS theories.
} 
Nonetheless, it is important to keep in mind that HS conformal theories are naturally higher derivative theories and for this reason violate unitarity, just as conformal gravity. This feature allows them to bypass the Coleman-Mandula theorem as well as other powerful no-go theorems in flat space. ${ }^{2}$ On the other hand it has been recently pointed out how asymptotically AdS solutions of Einstein gravity can be recovered from four derivative theories by choosing appropriate boundary conditions [34-36]. This provides some key hints about the role of the latter non-unitary theories in the context of AdS/CFT. Therefore, these features motivate a closer look at conformal HS theories and their properties.

Free Lagrangians and the corresponding wave equations involving massless Fronsdal fields and their variants have received considerable interest [37-47]. But the explicit form of the conformal wave operator for HS fields in curved spaces has not been worked out yet. ${ }^{3}$ The aim of this paper is to study free conformal higher spins actions and the corresponding wave operators on generic backgrounds. One of our goals is to discuss the factorization property of the conformal wave operator for HS fields generalizing previous result for spin 2. We have also been able to fix the conformal wave operator in $d=4$ for $s=3$ up to linear order in the Riemann tensor on generic Bach-flat backgrounds. As a byproduct of our analysis, we obtain the full conformal wave operator on (A)dS backgrounds in any dimension in a manifestly factorized form. Each factor turns out to be given by a two derivative operator. Their combined mass spectrum comprises the massless and partiallymassless points plus massive points in higher dimensions [5, 43, 55-61]. This provides additional evidence for previous conjectures made in $[48,62]$ and extends them. In addition, we also identify the Weyl tensor and its derivatives as the obstruction to factorization for spin $s>2$ on generic backgrounds. Furthermore, we rediscover the well known factorization of the conformal wave operator for spin 2 on Einstein backgrounds [3-6], and extend it to arbitrary dimensions.

The obstruction to factorization for $\operatorname{spin} s>2$ can be interpreted as a conformal reincarnation of the Aragone-Deser obstruction [63] for two derivative HS wave operators. Indeed, the crucial difference between spin 2 and HS fields is the explicit appearance of the Weyl tensor within the gauge variation of the generic two derivative operators. On the contrary, any contribution proportional to the Weyl tensor can be eliminated for spin 1 and 2 , making their wave operator factorizable.

Amongst other things we also develop a variant of the HS tractor calculus (see e.g [64] and references therein), that finds potentially useful applications to conformal HS fields. We believe that this formalism might provide a useful tool for addressing various problems with conformal higher spin fields, like for instance the extension of the present analysis to interactions and to the study of conformal HS algebras.

The organization of the paper is as follows. In section 2 we describe a convenient formalism that allows us to deal with conformal fields in a simplified way. In section 3 we test the consistency of a factorized conformal wave operator in generic backgrounds

\footnotetext{
${ }^{2}$ See e.g. [22] and references therein for a review of various no-go theorems and [33] for a stronger version of the Coleman-Mandula theorem in flat space.

${ }^{3}$ See [48] for some discussion of higher derivative theories in flat space, $[49,50]$ for some earlier discussion on conformal operators and [51-54] for selected math literature.
} 
studying the obstructions to factorization. In section 4 we give the spin 3 conformal wave operator on Bach-flat backgrounds up to linear order in the Riemann tensor. In section 5 we summarize our results and conclude. We have put additional material that includes a discussion about gauge fixing and some lower-spin examples in the appendices. Lastly, we have attached a Mathematica notebook containing independent checks as an ancillary file.

\section{Conformal higher spin fields}

Conformal higher spin fields $[8,13]$ can be defined at the linear level by demanding the following gauge invariance properties

$$
\begin{aligned}
\delta_{\xi} \varphi_{\mu_{1} \cdots \mu_{s}} & =\nabla_{\left(\mu_{1}\right.} \xi_{\left.\mu_{2} \cdots \mu_{s}\right)}, \\
\delta_{\alpha} \varphi_{\mu_{1} \cdots \mu_{s}} & =g_{\left(\mu_{1} \mu_{2}\right.} \alpha_{\left.\mu_{3} \cdots \mu_{s}\right)}
\end{aligned}
$$

No trace constraints on fields or gauge parameters are imposed. The above generalizes the linearized gauge invariance and rescaling invariance of conformal gravity. Indeed, for spin 2 equation (2.1b) describes linear dilatations (scale transformations). For higher spins, on top of the above transformations, one would in principle also need to consider also proper HS scale transformations of the form $\varphi_{\mu_{1} \cdots \mu_{s}} \rightarrow \Omega \varphi_{\mu_{1} \cdots \mu_{s}}$. But for the purpose of this paper it will not be necessary to impose this beforehand. Irrespectively, the wave operators we find turn out to be automatically invariant under these scale transformations.

We will now switch to an operator notation where fields are represented by generating functions,

$$
\varphi_{\mu_{1} \cdots \mu_{s}}(x) \rightarrow \varphi(x, u)=\frac{1}{s !} \varphi_{\mu_{1} \cdots \mu_{s}}(x) e_{a_{1}}^{\mu_{1}}(x) u^{a_{1}} \cdots e_{a_{s}}^{\mu_{s}}(x) u^{a_{s}}
$$

Here we have introduced a constant auxiliary tangent variable $u^{a}$. See appendix A for all our notational conventions and a brief introduction to the operator formalism. In the operator notation the gauge invariance properties (2.1) take the form

$$
\begin{aligned}
& \delta_{\xi} \varphi(x, u)=u \cdot \nabla \xi(x, u), \\
& \delta_{\alpha} \varphi(x, u)=u^{2} \alpha(x, u) .
\end{aligned}
$$

From this it follows that a conformal field can be regarded as an equivalence class of standard massless higher spin fields defined on the cone $u^{2} \sim 0$. This observation allows us to use so-called Thomas-D derivatives $\hat{\partial}_{u}$ in the auxiliary variable $u$. Again, see appendix A for more information.

We now summarize our results. We find the following manifestly factorized form of the spin $s$ conformal wave operator in $(\mathrm{A}) \mathrm{dS}_{d}$ :

$$
\mathcal{O}^{(s)}=\prod_{i=1}^{\frac{d}{2}-2+s}\left[\square-\frac{d-4+2 s}{i(d-3-i+2 s)} u \cdot \nabla \nabla \cdot \hat{\partial}_{u}+\Lambda[(i-s+1)(i-s-d+2)-s]\right],
$$


Similarly, the factorized spin 2 conformal wave operator on any Einstein background can be expressed as

$$
\mathcal{O}^{(2)}=\prod_{i=1}^{\frac{d}{2}}\left[\square-\frac{d}{i(d+1-i)} u \cdot \nabla \nabla \cdot \hat{\partial}_{u}+\Lambda[(i-1)(i-d)-2]+W_{\mu \nu \rho \sigma} u^{\mu} u^{\rho} \hat{\partial}_{u_{\nu}} \hat{\partial}_{u_{\rho}}\right] .
$$

The conformal wave operator for higher spins does not factorize on generic Einstein spaces, as we shall demonstrate in the next section.

\section{Factorization of conformal wave operators}

In this section we study the obstructions for a factorized conformal wave operator to be gauge invariant on generic backgrounds. Our soon to be disproved assumption is that the conformal wave operator factorizes into two-derivative operators on any Bach-flat background, or generalizations thereof in $d>4$. The existence of a conformal wave operator on Bach-flat backgrounds can be argued on the basis of the following two observations.

Firstly, an Aragone and Deser type of obstruction [63] cannot arise since the conformal coupling with gravity has the same number of derivatives as the kinetic term at any order in the spin $s$ field. In particular any coupling of the type $s-s-2-\ldots-2$ involving $n$ spin two fields and two spin $s$ fields must involve $2 s+d-4$ derivatives. This type of obstruction appears for two derivative operators like the Fronsdal operator because the corresponding gravitational couplings are higher derivative [33, 65, 66].

Secondly, any possible tadpoles (i.e. vertices linear in the higher spin field) can be removed. In principle one might be forced to add them, but they can always be integrated by parts into a non-linear equation for the metric. In $d=4$ this equation will involve the Bach tensor, although in general it will become a higher derivative condition for the metric containing $2 s+d-4$ derivatives. For this reason it will be compatible with, if not equivalent to, the conformal gravity equations of motion.

We will come back to constructing a conformal invariant operators on generic backgrounds in section 4. But first we will concentrate on an Ansatz that is explicitly factorized, with the aim of identifying the obstruction to its gauge invariance. The non-existence of a factorized solution in general will not imply the non-existence of the full operator. In fact, we expect to the full operator to exist for any spin on generic conformal manifolds for the reason mentioned above.

\subsection{Ansatz}

A $(2 s+d-4)$-derivative factorized Ansatz for the conformal spin $s$ wave operator can be written as

$$
\mathcal{O}^{(s)}=\prod_{i=1}^{\frac{d}{2}-2+s} \mathcal{F}_{i}
$$

where $\mathcal{F}_{i}$ is the most general Ansatz for a two derivative operator:

$$
\mathcal{F}_{i}=\square+\alpha_{i} u \cdot \nabla \nabla \cdot \hat{\partial}_{u}+\beta_{i} \Lambda+\gamma_{i} R_{\mu \nu \rho \sigma}^{\Lambda} u^{\mu} u^{\rho} \hat{\partial}_{u_{\nu}} \hat{\partial}_{u_{\sigma}}+\delta_{i} R_{\mu \nu}^{\Lambda} u^{\mu} \hat{\partial}_{u_{\nu}}+\sigma_{i} R^{\Lambda} .
$$


Here we have defined $R_{\mu \nu \rho \sigma}^{\Lambda}=R_{\mu \nu \rho \sigma}-\Lambda\left(g_{\mu \rho} g_{\nu \sigma}-g_{\nu \rho} g_{\mu \sigma}\right)$, and similarly for the Ricci tensor (see also appendix A). On Einstein backgrounds this simplifies to

$$
\mathcal{F}_{i}^{\mathrm{E}}=\square+\alpha_{i} u \cdot \nabla \nabla \cdot \hat{\partial}_{u}+\beta_{i} \Lambda+\gamma_{i} W_{\mu \nu \rho \sigma} u^{\mu} u^{\rho} \hat{\partial}_{u_{\nu}} \hat{\partial}_{u_{\sigma}},
$$

where $W_{\mu \nu \rho \sigma}$ is the Weyl tensor.

For the purpose of enforcing gauge invariance of the full operator it is useful to compute the gauge transformation of the generic two-derivative operator $\mathcal{F}_{i}$. It reads

$$
\begin{aligned}
\mathcal{F} u \cdot \nabla= & u \cdot \nabla\left[(1+\alpha) \square+\alpha\left(1-\frac{2}{h}\right) u \cdot \nabla \nabla \cdot \hat{\partial}_{u}\right. \\
& \left.+\Lambda\left\{\beta+u \cdot \hat{\partial}_{u}+1+\left(\alpha u \cdot \hat{\partial}_{u}+1\right)\left(u \cdot \hat{\partial}_{u}+d-2\right)\right\}\right] \\
& -\alpha\left(u \cdot \nabla R_{\mu \nu \rho \sigma}^{\Lambda}\right) u^{\mu} u^{\rho} \hat{\partial}_{u_{\nu}} \hat{\partial}_{u_{\sigma}}+(\gamma-\alpha) R_{\mu \nu \rho \sigma}^{\Lambda} u \cdot \nabla u^{\mu} u^{\rho} \hat{\partial}_{u_{\nu}} \hat{\partial}_{u_{\sigma}} \\
& +2(\gamma-1) R_{\mu \nu \rho \sigma}^{\Lambda} u^{\mu} \nabla^{\nu} u^{\rho} \hat{\partial}_{u_{\sigma}}+(1+\alpha)\left(u \cdot \nabla R_{\mu \nu}^{\Lambda}\right) u^{\mu} \hat{\partial}_{u_{\nu}} \\
& +(\alpha+\delta) R_{\mu \nu}^{\Lambda} u \cdot \nabla u^{\mu} \hat{\partial}_{u_{\nu}}+(1+\delta) R_{\mu \nu}^{\Lambda} u^{\mu} \nabla^{\nu}-u^{\mu} u^{\nu}\left(\nabla \cdot \hat{\partial}_{u} R_{\mu \nu}^{\Lambda}\right) \\
& -\frac{2}{h-2}(\gamma+\delta) R_{\mu \nu}^{\Lambda} u^{\mu} u^{\nu} \nabla \cdot \hat{\partial}_{u}+\sigma R^{\Lambda} u \cdot \nabla .
\end{aligned}
$$

First of all, the structure of the gauge variation illustrates an important difference between spin-2 and higher spins. This is due to the appearance of terms proportional to the full Riemann tensor and its derivatives, for instance

$$
\left(u \cdot \nabla R_{\mu \nu \rho \sigma}^{\Lambda}\right) u^{\mu} u^{\rho} \hat{\partial}_{u_{\nu}} \hat{\partial}_{u_{\sigma}} .
$$

This term, being cubic in the auxiliary variable $u$, appears only for spin $s \geq 3$. This is actually a reincarnation of the same feature pointed out by Aragone and Deser [63] in the context of Fronsdal fields.

The above gauge variation can be used to recursively compute the gauge variation of the factorized Ansatz (3.1). Using the notation

$$
\mathcal{F}_{i} u \cdot \nabla=u \cdot \nabla \tilde{\mathcal{F}}_{i}+\mathcal{X}_{i}
$$

we get

$$
\left(\mathcal{F}_{1} \cdots \mathcal{F}_{n}\right) u \cdot \nabla=u \cdot \nabla\left(\tilde{\mathcal{F}}_{1} \cdots \tilde{\mathcal{F}}_{n}\right)+\mathcal{X}^{(n)},
$$

where $\mathcal{X}^{(n)}$ is recursively defined as

$$
\mathcal{X}^{(n)}=\mathcal{X}_{1} \tilde{\mathcal{F}}_{2} \ldots \tilde{\mathcal{F}}_{n}+\mathcal{F}_{1} \mathcal{X}^{(n-1)}=\sum_{k=1}^{n} \mathcal{F}_{1} \ldots \mathcal{F}_{k-1} \mathcal{X}_{k} \tilde{\mathcal{F}}_{k+1} \ldots \tilde{\mathcal{F}}_{n}
$$

It is then straightforward to see that gauge invariance for the spin $s$ wave operator implies the condition

$$
u \cdot \nabla\left(\tilde{\mathcal{F}}_{\frac{d}{2}-2+s} \cdots \tilde{\mathcal{F}}_{1}\right)+\mathcal{X}^{\left(\frac{d}{2}-2+s\right)}=0
$$




\subsection{Arbitrary spins on AdS backgrounds}

The coefficients $\alpha$ and $\beta$ enter the Riemann-independent part of the Ansatz (3.2). Hence, in order to fix them it is sufficient to look at the zeroth order in the $R^{\Lambda}$ tensors. This corresponds to the case of (A)dS, which we are going to consider in detail in this section. The equation (3.9) will simplify and will admit an iterative structure, which is crucial for factorization.

At zeroth order in Riemann tensors we have $\mathcal{X}_{i} \sim 0$, and end up with the following equation for the $i^{\text {th }}$ factor in the Ansatz:

$$
\begin{aligned}
\left(\nabla \cdot \hat{\partial}_{u}\right)^{i-1}[ & \left(1+\alpha_{i}\right) \square+\alpha_{i}\left(1-\frac{2}{h}\right) u \cdot \nabla \nabla \cdot \hat{\partial}_{u} \\
& \left.+\Lambda\left(\beta_{i}+u \cdot \hat{\partial}_{u}+1+\left(\alpha_{i} u \cdot \hat{\partial}_{u}+1\right)\left(u \cdot \hat{\partial}_{u}+d-2\right)\right)\right] \sim\left(\nabla \cdot \hat{\partial}_{u}\right)^{i}
\end{aligned}
$$

This recursively ensures that all terms proportional to $\left(\nabla \cdot \hat{\partial}_{u}\right)^{i-1}$ vanish. In principle we should also impose that no higher divergence is generated, but this condition turns out to be automatically satisfied if the number of derivatives is chosen to be $2 s+d-4$. We will now fix all $\alpha$ 's and $\beta$ 's by solving linear equations. We begin with observing that

$$
\left(\nabla \cdot \hat{\partial}_{u}\right)^{k} \tilde{\mathcal{F}}_{i}=\left(\nabla \cdot \hat{\partial}_{u}\right)^{k-i}\left[a_{i} \square+b_{i} u \cdot \nabla \nabla \cdot \hat{\partial}_{u}+c_{i} \Lambda\right]\left(\nabla \cdot \hat{\partial}_{u}\right)^{i}+\mathcal{O}\left(R^{\Lambda}\right),
$$

where the coefficients satisfy the following recursion relations:

$$
\begin{aligned}
& a_{j}=a_{j-1}+b_{j-1}, \\
& b_{j}=b_{j-1}\left(1-\frac{1}{\frac{d}{2}-2+s-j}\right), \\
& c_{j}=c_{j-1}+b_{j-1}(s-j-1)(s-j+d-3)+a_{j-1}(2(s-j)+d-3) .
\end{aligned}
$$

These recursion relations have boundary conditions

$$
\begin{aligned}
& a_{0}=1+\alpha_{i}, \\
& b_{0}=\alpha_{i}\left(1-\frac{1}{\frac{d}{2}-2+s}\right), \\
& c_{0}=\beta_{i}+s+\left(\alpha_{i}(s-1)+1\right)(s+d-3) .
\end{aligned}
$$

The solution to the first two recursion relations reads:

$$
\begin{aligned}
& a_{j}=1+\alpha_{i}\left[1+j\left(1-\frac{1+j}{d-4+2 s}\right)\right], \\
& b_{j}=\alpha_{i}\left(1-\frac{1+j}{\frac{d}{2}-2+s}\right) .
\end{aligned}
$$

We do not write the solution for $c_{i}$ since it is rather cumbersome and enters only intermediate steps of the computation. We can now enforce gauge invariance by recursively 
demanding that terms proportional to a divergence vanish in the gauge variation. We end up with

$$
\begin{aligned}
a_{i-1}\left(\alpha_{i}\right) & =0, \\
c_{i-1}\left(\alpha_{i}, \beta_{i}\right) & =0 .
\end{aligned}
$$

The solution to these equations reads

$$
\begin{aligned}
\alpha_{i} & =-\frac{1}{1+(i-1)\left(1-\frac{i}{d-4+2 s}\right)}, \\
\beta_{i} & =(i-s+1)(i-s-d+2)-s .
\end{aligned}
$$

And so the conformal wave operator on (A)dS takes the form

$$
\mathcal{O}^{(s)}=\prod_{i=1}^{\frac{d}{2}-2+s}\left[\square-\frac{d-4+2 s}{i(d-3-i+2 s)} u \cdot \nabla \nabla \cdot \hat{\partial}_{u}+\Lambda((i-s+1)(i-s-d+2)-s)\right] .
$$

Finally, the action which has $\mathcal{O}^{(s)} \varphi_{(s)}=0$ as an equation of motion reads

$$
S^{(s)}=\left.\frac{1}{2} \int d^{d} x \sqrt{-g} e^{\hat{\partial}_{u_{1}} \cdot \hat{\partial}_{u_{2}}} \varphi_{(s)}\left(u_{1}\right) \mathcal{O}^{(s)} \varphi_{(s)}\left(u_{2}\right)\right|_{u_{i}=0} .
$$

This reproduces the correct equations of motion because the operator $\mathcal{O}$ is automatically self-adjoint up to total derivatives.

It is worth pointing out that the coefficients $\beta$ precisely match the masses associated with the partially massless points for spin $s$, plus some discrete massive points in $d>4$. This is in agreement with previous conjectures on conformal HS wave operators [48, 62]. This implies in turn that the part of the conformal operator that is not proportional to divergences or traces has the form

$$
\mathcal{O} \sim \prod_{i=0}^{\frac{d}{2}-3+s}[\square+\Lambda((i-s+2)(i-s-d-3)-s)] .
$$

In appendix $\mathrm{C}$ we show that terms involving divergences can be set to zero by choosing a convenient gauge.

Before concluding this section it is important to comment that strictly speaking the above discussion is sufficient to determine the full conformal spin $s$ operator on (A)dS only in $d=4$, where the number of derivatives required by scale invariance is $2 s$. In higher even dimensions the first $s$ factors have to be the same as above but the next $\frac{d}{2}-2$ factors are not constrained by gauge invariance and one would need to analyze conformal invariance more closely. Notice that conformal invariance,

$$
\delta g_{\mu \nu}=\Omega(x)^{2} g_{\mu \nu}
$$

is not easy to prove due to the generically complicated transformation properties of covariant derivatives. However, the condition of gauge invariance at the operator level,

$$
\mathcal{O}^{(s)} u \cdot \nabla=0
$$


is strong enough to completely fix the wave operator in any dimension. Loosely speaking, operator gauge invariance means that the kernel of $\mathcal{O}^{(s)} u \cdot \nabla$ is enlarged from the HS gauge parameters $\xi$ to arbitrary homogeneous functions of $u .{ }^{4}$ Moreover, we have also checked in appendix $\mathrm{C}$ that the factorization Ansatz does not play any role and one can arrive at analogous results starting from a more general Ansatz. One can then argue, and check with examples (see appendix D), that the stronger gauge invariance condition (3.21), implies conformal invariance when the operator $\mathcal{O}_{s}$ is defined on the equivalence classes (A.8). In $d=4$ the crucial simplification is that the operator gauge invariance and the usual gauge invariance conditions coincide.

From a group-theoretical perspective the operator gauge invariance implies also that the pattern of masses follows a very simple relation,

$$
E=d+s-3-i \text { for } 0 \leq i \leq \frac{d}{2}-3+s .
$$

This is nothing but the continuation of the pattern of the (partially-)massless points, $0 \leq i \leq s-1$, to massive points. Furthermore, it is what is expected from the decomposition of a representation of the conformal algebra with respect to the (A)dS subalgebra [62]. It is remarkable that the above requirements can be recast in terms of a usual gauge invariance condition extended to the operator level. For these reasons, it might provide a useful tool to control conformal invariance (3.20).

So far we have been able to completely fix the conformal wave operator on (A)dS. In the following we will analyze the same problem in generic backgrounds. We shall first consider the spin-2 case in more detail, and then address the higher spin problem.

\subsection{Spin 2 on generic backgrounds}

The spin 2 case is special with respect to its higher spin cousins because the commutation relations (A.14) simplify. In particular, terms of order $u^{3}$ or $\hat{\partial}_{u}^{3}$ in the conformal operator as well as terms of order $\hat{\partial}_{u}^{2}$ in gauge variation drop out. The gauge variation of a single $\mathcal{F}(3.4)$ simplifies to

$$
\begin{aligned}
\mathcal{F} u \cdot \nabla= & u \cdot \nabla\left[(1+\alpha) \square+\alpha\left(1-\frac{2}{h}\right) u \cdot \nabla \nabla \cdot \hat{\partial}_{u}\right. \\
& \left.+\Lambda\left(\beta+u \cdot \hat{\partial}_{u}+1+\left(\alpha u \cdot \hat{\partial}_{u}+1\right)\left(u \cdot \hat{\partial}_{u}+d-2\right)\right)\right] \\
& +2(\gamma-1) R_{\mu \nu \rho \sigma}^{\Lambda} u^{\mu} \nabla^{\nu} u^{\rho} \hat{\partial}_{u_{\sigma}}+(1+\alpha)\left(u \cdot \nabla R_{\mu \nu}^{\Lambda}\right) u^{\mu} \hat{\partial}_{u_{\nu}} \\
& +(\alpha+\delta) R_{\mu \nu}^{\Lambda} u \cdot \nabla u^{\mu} \hat{\partial}_{u_{\nu}}-u^{\mu} u^{\nu}\left(\nabla \cdot \hat{\partial}_{u} R_{\mu \nu}^{\Lambda}\right)+(1+\delta) R_{\mu \nu}^{\Lambda} u^{\mu} \nabla^{\nu} \\
& -\frac{2}{h-2}(\gamma+\delta) R_{\mu \nu}^{\Lambda} u^{\mu} u^{\nu} \nabla \cdot \hat{\partial}_{u}+\sigma R^{\Lambda} u \cdot \nabla .
\end{aligned}
$$

This enables us to eliminate all instances of the Riemann tensor by simply choosing $\gamma=1$. This very simple observation is sufficient to ensure that the factorized Ansatz works on any Einstein background.

\footnotetext{
${ }^{4}$ Enlarging the domain of formal generating functions to distributions has also been done in [67].
} 
However, it should be clear from the argument itself that this simplification is nongeneric. For completeness and to underline the non-generic nature, let us analyze the factorization of the conformal spin 2 operator on general backgrounds more closely. Taking the solution (3.16) for $\alpha$ 's and $\beta$ 's obtained in the previous section into account, the gaugeinvariance condition reads in $d=4$

$$
\mathcal{X}_{1} \tilde{\mathcal{F}}_{2}+\mathcal{F}_{1} \mathcal{X}_{2}=0
$$

Here we have

$$
\begin{aligned}
\mathcal{X}_{i}= & +\left(1+\alpha_{i}\right)\left(u \cdot \nabla R_{\mu \nu}^{\Lambda}\right) u^{\mu} \hat{\partial}_{u_{\nu}}+2\left(\gamma_{i}-1\right) R_{\mu \nu \rho \sigma}^{\Lambda} u^{\mu} \nabla^{\nu} u^{\rho} \hat{\partial}_{u_{\sigma}} \\
& +\left(\alpha_{i}+\delta_{i}\right) R_{\mu \nu}^{\Lambda} u \cdot \nabla u^{\mu} \hat{\partial}_{u_{\nu}} \quad+\left(1+\delta_{i}\right) R_{\mu \nu}^{\Lambda} u^{\mu} \nabla^{\nu}-u^{\mu} u^{\nu}\left(\nabla \cdot \hat{\partial}_{u} R_{\mu \nu}^{\Lambda}\right) \\
& -\frac{2}{h-2}\left(\gamma_{i}+\delta_{i}\right) R_{\mu \nu}^{\Lambda} u^{\mu} u^{\nu} \nabla \cdot \hat{\partial}_{u}+\sigma_{i} R^{\Lambda} u \cdot \nabla \\
\mathcal{F}_{1}= & \square-u \cdot \nabla \nabla \cdot \hat{\partial}_{u}-2 \Lambda \\
\tilde{\mathcal{F}}_{2}= & \frac{1}{3} \square-\frac{1}{3} u \cdot \nabla \nabla \cdot \hat{\partial}_{u}-\Lambda .
\end{aligned}
$$

The terms linear in $R_{\mu \nu \rho \sigma}^{\Lambda}$ without any divergence are

$$
2 R_{\mu \nu \rho \sigma}^{\Lambda} u^{\mu} \nabla^{\nu} u^{\rho} \hat{\partial}_{u_{\sigma}}\left[\left(\gamma_{1}-1\right)\left(\frac{1}{3} \square-\Lambda\right)+\left(\gamma_{2}-1\right)(\square-2 \Lambda)\right] .
$$

It is easy to see that the only solution to gauge invariance is $\gamma_{i}=1$, which eliminates any instance of the Riemann tensor in the gauge variation. In order to study the obstructions related to $R_{\mu \nu}^{\Lambda}$ it is useful to first concentrate on the terms that do not involve any derivative of $R_{\mu \nu}^{\Lambda}$. Thus for the moment we will set $\nabla_{\alpha} R_{\mu \nu} \sim 0$ and, as a consequence of the Bianchi identity, $R^{\Lambda} \sim 0$ (i.e. the non-constant part of the Ricci scalar vanishes). Dropping terms proportional to divergences for simplicity, we get the following gauge variation:

$$
\begin{aligned}
\mathcal{O}^{(2)} u \cdot \nabla \sim & +\left[\left(\delta_{1}+1\right) R_{\mu \nu}^{\Lambda} u^{\mu} \nabla^{\nu}+\left(\delta_{1}-1\right) R_{\mu \nu}^{\Lambda} u \cdot \nabla u^{\mu} \hat{\partial}_{u_{\nu}}\right]\left[\frac{1}{3} \square-\Lambda\right] \\
& -\frac{1}{6}\left(\delta_{1}+1\right) R_{\mu \nu}^{\Lambda} u^{\mu} u^{\nu} \nabla \cdot \hat{\partial}_{u} \square \\
& +[\square-2 \Lambda]\left[\left(1+\delta_{2}\right) R_{\mu \nu}^{\Lambda} u^{\mu} \nabla^{\nu}+\left(\alpha_{2}+\delta_{2}\right) R_{\mu \nu}^{\Lambda} u \cdot \nabla u^{\mu} \hat{\partial}_{u_{\nu}}\right] \\
& -u \cdot \nabla \nabla \cdot \hat{\partial}_{u}\left[\left(1+\delta_{2}\right) R_{\mu \nu}^{\Lambda} u^{\mu} \nabla^{\nu}+\left(\alpha_{2}+\delta_{2}\right) R_{\mu \nu}^{\Lambda} u \cdot \nabla u^{\mu} \hat{\partial}_{u_{\nu}}\right] .
\end{aligned}
$$

Keeping only terms of the order $\left(R^{\Lambda}\right)^{2}$ and commuting all boxes until they act on the gauge parameter while dropping divergences, we obtain:

$$
\begin{aligned}
-\frac{1}{6}\left(\delta_{1}+1\right) & R_{\mu \nu}^{\Lambda} u^{\mu} u^{\nu} R_{\mu \nu}^{\Lambda} \nabla^{\mu} \hat{\partial}_{u_{\nu}} \\
& \left(1+\delta_{2}\right)\left[-2 R_{\mu \alpha}^{\Lambda} R_{\nu \rho \sigma}^{\Lambda \alpha} u^{\mu} \nabla^{\nu} u^{\rho} \hat{\partial}_{u_{\sigma}}+R_{\mu \alpha}^{\Lambda} R_{\nu}^{\Lambda \alpha} u^{\mu} \nabla^{\nu}\right] \\
& +\left(\alpha_{2}+\delta_{2}\right)\left[-2 R_{\mu \nu \rho \alpha}^{\Lambda} R^{\Lambda \alpha}{ }_{\sigma} u^{\mu} \nabla^{\nu} u^{\rho} \hat{\partial}_{u_{\sigma}}+R_{\mu \nu}^{\Lambda} u^{\mu} \nabla^{\nu} R_{\rho \sigma}^{\Lambda} u^{\rho} \hat{\partial}_{u_{\sigma}}\right] \\
& -\left(\alpha_{2}+\delta_{2}\right) u \cdot \nabla R_{\mu \beta}^{\Lambda} R_{\nu}^{\Lambda \beta} u_{\nu}^{\mu} \hat{\partial}_{u_{\nu}} .
\end{aligned}
$$


This cannot be set to zero by tuning the free coefficients, which implies $R_{\mu \nu}^{\Lambda}$ is an obstruction to factorization in the spin two case. This concludes the proof that factorization of the spin-2 conformal wave operator is possible only on Einstein backgrounds. As we have seen above its form is remarkably simple and can be written as

$$
\begin{aligned}
\mathcal{O}^{(2)}=\left(\square-u \cdot \nabla \nabla \cdot \hat{\partial}_{u}-2 \Lambda\right. & \left.+W_{\mu \nu \rho \sigma} u^{\mu} u^{\rho} \hat{\partial}_{u_{\nu}} \hat{\partial}_{u_{\sigma}}\right) \\
& \times\left(\square-\frac{2}{3} u \cdot \nabla \nabla \cdot \hat{\partial}_{u}-4 \Lambda+W_{\mu \nu \rho \sigma} u^{\mu} u^{\rho} \hat{\partial}_{u_{\nu}} \hat{\partial}_{u_{\sigma}}\right) .
\end{aligned}
$$

On more general conformal manifolds factorization is not possible.

The above discussion generalizes readily to any dimension, upon which we get the following manifestly factorized form of the spin 2 conformal wave operator:

$$
\mathcal{O}^{(2)}=\prod_{i=1}^{\frac{d}{2}}\left[\square-\frac{d}{i(d+1-i)} u \cdot \nabla \nabla \cdot \hat{\partial}_{u}+\Lambda[(i-1)(i-d)-2]+W_{\mu \nu \rho \sigma} u^{\mu} u^{\rho} \hat{\partial}_{u_{\nu}} \hat{\partial}_{u_{\sigma}}\right],
$$

Before concluding this section, let us point out that the above result is the unique operator that factorizes, and it reduces to our previous result (2.4) upon restricting to (A)dS backgrounds. If the factorization requirement is dropped more conformal operators can be found, e.g. by linearizing the conformal invariant densities of [68-71]. However, all but one of these densities vanish when linearized on (A)dS backgrounds as they consist of more than two Weyl tensors. See also section D.2 for an example of this for $d=6$.

We will now proceed to the higher spin cases. Due to the generic nature of the obstructions we found for spin 2, we will restrict our attention to Einstein manifolds in what follows.

\subsection{Higher spins on Einstein backgrounds}

We will now consider arbitrary spins on Einstein backgrounds, and consequently set $R_{\mu \nu}^{\Lambda}$ to zero. Upon doing so, the commutation relations simplify drastically and the gauge variation of a single $\mathcal{F}$, equation (3.4), becomes

$$
\begin{aligned}
\mathcal{F} u \cdot \nabla & =u \cdot \nabla\left[(1+\alpha) \square+\alpha\left(1-\frac{2}{h}\right) u \cdot \nabla \nabla \cdot \hat{\partial}_{u}\right. \\
& \left.+\Lambda\left(\beta+u \cdot \hat{\partial}_{u}+1+\left(\alpha u \cdot \hat{\partial}_{u}+1\right)\left(u \cdot \hat{\partial}_{u}+d-2\right)\right)\right] \\
& -\alpha\left(u \cdot \nabla W_{\mu \nu \rho \sigma}\right) u^{\mu} u^{\rho} \hat{\partial}_{u_{\nu}} \hat{\partial}_{u_{\sigma}}+(\gamma-\alpha) W_{\mu \nu \rho \sigma} u \cdot \nabla u^{\mu} u^{\rho} \hat{\partial}_{u_{\nu}} \hat{\partial}_{u_{\sigma}} \\
& +2(\gamma-1) W_{\mu \nu \rho \sigma} u^{\mu} \nabla^{\nu} u^{\rho} \hat{\partial}_{u_{\sigma}} .
\end{aligned}
$$

To analyze if the Weyl tensor is an obstruction it is useful to drop all of its derivatives and set

$$
\begin{aligned}
\nabla_{\alpha} W_{\mu \nu \rho \sigma} & \sim 0, \\
{\left[\nabla_{\beta}, \nabla_{\alpha}\right] W_{\mu \nu \rho \sigma} } & \sim 0 .
\end{aligned}
$$


We can then rewrite equation (3.33) as

$$
\begin{aligned}
\mathcal{F} u \cdot \nabla \sim u \cdot \nabla & {\left[(1+\alpha) \square+\alpha\left(1-\frac{2}{h}\right) u \cdot \nabla \nabla \cdot \hat{\partial}_{u}\right.} \\
& +\Lambda\left(\beta+u \cdot \hat{\partial}_{u}+1+\left(\alpha u \cdot \hat{\partial}_{u}+1\right)\left(u \cdot \hat{\partial}_{u}+d-2\right)\right) \\
& \left.+(\gamma-\alpha) W_{\mu \nu \rho \sigma} u^{\mu} u^{\rho} \hat{\partial}_{u_{\nu}} \hat{\partial}_{u_{\sigma}}\right] \\
+ & 2(\gamma-1) W_{\mu \nu \rho \sigma} u^{\mu} \nabla^{\nu} u^{\rho} \hat{\partial}_{u_{\sigma}} .
\end{aligned}
$$

The gauge variation of the factorized Ansatz becomes

$$
\delta \mathcal{O}^{(s)}=u \cdot \nabla \tilde{\mathcal{F}}_{1} \ldots \tilde{\mathcal{F}}_{\frac{d}{2}-2+s}+\sum_{k=1}^{\frac{d}{2}-2+s} \mathcal{F}_{1} \ldots \mathcal{F}_{k-1} \mathcal{X}_{k} \tilde{\mathcal{F}}_{k+1} \ldots \tilde{\mathcal{F}}_{\frac{d}{2}-2+s}
$$

where

$$
\begin{aligned}
\tilde{\mathcal{F}}_{i}= & \left(1+\alpha_{i}\right) \square+\alpha_{i}\left(1-\frac{2}{h}\right) u \cdot \nabla \nabla \cdot \hat{\partial}_{u} \\
& +\Lambda\left(\beta_{i}+u \cdot \hat{\partial}_{u}+1+\left(\alpha_{i} u \cdot \hat{\partial}_{u}+1\right)\left(u \cdot \hat{\partial}_{u}+d-2\right)\right) \\
& +\left(\gamma_{i}-\alpha_{i}\right) W_{\mu \nu \rho \sigma} u^{\mu} u^{\rho} \hat{\partial}_{u_{\nu}} \hat{\partial}_{u_{\sigma}} \\
\mathcal{F}_{i}= & \square+\alpha_{i} u \cdot \nabla \nabla \cdot \hat{\partial}_{u}+\beta_{i} \Lambda+\gamma_{i} W_{\mu \nu \rho \sigma} u^{\mu} u^{\rho} \hat{\partial}_{u_{\nu}} \hat{\partial}_{u_{\sigma}} \\
\mathcal{X}_{k}= & 2\left(\gamma_{k}-1\right) W_{\mu \nu \rho \sigma} u^{\mu} \nabla^{\nu} u^{\rho} \hat{\partial}_{u_{\sigma}} .
\end{aligned}
$$

We can now concentrate on terms involving the Weyl tensor via the combination

$$
\left(W_{\mu \nu \rho \sigma} u^{\mu} u^{\rho} \hat{\partial}_{u_{\nu}} \hat{\partial}_{u_{\sigma}}\right)^{m} .
$$

These include terms proportional to powers of the Weyl tensor and the gauge parameter $\xi$,

$$
W_{\mu_{1} \nu_{1}}^{\alpha_{1} \beta_{1}} W_{\alpha_{1} \beta_{1}}^{\alpha_{2} \beta_{2}} \cdots W_{\alpha_{m} \beta_{m}}^{\rho_{1} \sigma_{1}} \xi_{\rho_{1} \sigma_{1} \ldots}
$$

and are non vanishing upon setting the derivatives of the Weyl tensor to zero. Moreover, they can arise only from the first contribution to the gauge variation. For this reason they need to vanish identically, so we are forced to impose the following condition:

$$
\gamma_{i}=\alpha_{i}, \quad \forall i
$$

Notice that we have used the defining properties of the $\alpha$ 's and $\beta$ 's in eq. (3.11) to simplify the terms involving divergences. However, when we now shift our attention to terms that involve the Weyl tensor via the combination

$$
\left(W_{\mu \nu \rho \sigma} u^{\mu} u^{\rho} \hat{\partial}_{u_{\nu}} \hat{\partial}_{u_{\sigma}}\right)^{m-1} W_{\mu \nu \rho \sigma} u^{\mu} \nabla^{\nu} u^{\rho} \hat{\partial}_{u_{\sigma}},
$$


we see that they do not vanish for covariantly constant Weyl tensors. Thus gauge invariance also requires

$$
\gamma_{i}=1, \quad \forall i
$$

The above clash of the gauge invariance condition identifies these particular Weyl tensor combinations, and hence generically the Weyl tensor, as the generic obstruction to factorization for the spin $s$ conformal wave operator on Einstein backgrounds. Moreover, we can also identify the first derivative of the Weyl tensor as an independent obstruction to factorization. This can be seen from (3.33) by looking at the contributions proportional to $\alpha\left(u \cdot \nabla W_{\mu \nu \rho \sigma} u^{\mu} u^{\rho} \hat{\partial}_{u_{\nu}} \hat{\partial}_{u_{\sigma}}\right)$, since none of the $\alpha$ 's is vanishing.

We have performed various independent checks of the above computations explicitly with the help of Mathematica. We have attached the corresponding notebook to this paper where the explicit spin 3 wave operator has been constructed up linear order in the Riemann tensor. In the next section we briefly summarize the contents of the notebook.

\section{Spin 3 wave operator on Bach-flat backgrounds}

With the help of Mathematica we have worked out the explicit form of the unique spin 3 conformal wave operator in $d=4$ up to linear terms in the Riemann tensor on Bach-flat backgrounds. We have done this by simply listing all possible contractions and constructing a gauge invariant Ansatz out of those. As expected from our arguments in section 3, we did not find any obstruction.

Furthermore, we also confirmed the invariance of the wave operator under Weyl rescalings of the metric (3.20). Remarkably, this turned out to be automatically the case after imposing gauge invariance under (2.1).

Even at linear order in Riemann tensors, the wave operator is rather unwieldy, consisting of roughly 200 terms. Its full form can be found in the attached notebook. Here we present the wave operator on Ricci flat backgrounds. It reads:

$$
\begin{aligned}
& \mathcal{O}_{\mu \nu \rho}^{(3)}(\varphi)=-\frac{21}{10} \nabla_{\mu \tau} R_{\nu \alpha \rho \beta} \nabla^{\sigma}{ }_{\sigma} \hat{\varphi}^{\tau \alpha \beta}-\frac{7}{10} \nabla_{\nu \alpha} R_{\rho \sigma \tau \beta} \nabla^{\sigma \tau} \hat{\varphi}_{\mu}{ }^{\alpha \beta}+\frac{182}{25} \nabla_{\sigma \tau} R_{\nu \alpha \rho \beta} \nabla^{\sigma \tau} \hat{\varphi}_{\mu}{ }^{\alpha \beta} \\
& -\frac{49}{25} \nabla_{\mu \nu} R_{\rho \alpha \tau \beta} \nabla^{\sigma \tau} \hat{\varphi}_{\sigma}{ }^{\alpha \beta}-\frac{49}{25} \nabla_{\mu \tau} R_{\nu \alpha \rho \beta} \nabla^{\sigma \tau} \hat{\varphi}_{\sigma}{ }^{\alpha \beta}-7 \nabla_{\mu \alpha} R_{\nu \tau \rho \beta} \nabla^{\sigma \tau} \hat{\varphi}_{\sigma}{ }^{\alpha \beta} \\
& -\frac{259}{25} \nabla_{\mu}{ }^{\sigma} \hat{\varphi}^{\tau \alpha \beta} \nabla_{\tau \sigma} R_{\nu \alpha \rho \beta}-\frac{84}{25} \nabla_{\mu}{ }^{\sigma} \hat{\varphi}^{\tau \alpha \beta} \nabla_{\tau \alpha} R_{\nu \sigma \rho \beta}+\frac{721}{50} \nabla^{\sigma \tau} \hat{\varphi}_{\mu}{ }^{\alpha \beta} \nabla_{\alpha \tau} R_{\nu \sigma \rho \beta} \\
& -\frac{161}{50} \nabla^{\sigma \tau} \hat{\varphi}_{\mu}{ }^{\alpha \beta} \nabla_{\alpha \beta} R_{\nu \sigma \rho \tau}-\frac{21}{5} \nabla_{\mu}{ }^{\sigma} \hat{\varphi}_{\sigma}{ }^{\tau \alpha} \nabla^{\beta}{ }_{\beta} R_{\nu \tau \rho \alpha}+\frac{252}{25} \nabla^{\sigma \tau} \hat{\varphi}_{\mu \sigma}{ }^{\alpha} \nabla^{\beta}{ }_{\beta} R_{\nu \tau \rho \alpha} \\
& -\frac{35}{2} \nabla^{\sigma \tau} \hat{\varphi}_{\mu \nu}{ }^{\alpha} \nabla^{\beta}{ }_{\beta} R_{\rho \sigma \tau \alpha}+\frac{343}{50} \nabla_{\mu}{ }^{\sigma} \hat{\varphi}_{\nu}{ }^{\tau \alpha} \nabla^{\beta}{ }_{\beta} R_{\rho \tau \sigma \alpha}-\frac{7}{5} \nabla_{\tau} R_{\rho \alpha \sigma \beta} \nabla_{\mu \nu}{ }^{\sigma} \hat{\varphi}^{\tau \alpha \beta} \\
& +\frac{7}{50} \nabla^{\sigma} \hat{\varphi}^{\tau \alpha \beta} \nabla_{\mu \nu \tau} R_{\rho \alpha \sigma \beta}-\frac{42}{5} \nabla_{\tau} R_{\nu \alpha \rho \beta} \nabla_{\mu}^{\sigma}{ }_{\sigma} \hat{\varphi}^{\tau \alpha \beta}+\frac{161}{50} \nabla_{\rho} R_{\sigma \alpha \tau \beta} \nabla_{\mu}{ }^{\sigma \tau} \hat{\varphi}_{\nu}^{\alpha \beta} \\
& +\frac{399}{50} \nabla_{\tau} R_{\rho \alpha \sigma \beta} \nabla_{\mu}{ }^{\sigma \tau} \hat{\varphi}_{\nu}{ }^{\alpha \beta}+\frac{441}{50} \nabla_{\alpha} R_{\rho \sigma \tau \beta} \nabla_{\mu}{ }^{\sigma \tau} \hat{\varphi}_{\nu}{ }^{\alpha \beta}-7 \nabla_{\nu} R_{\rho \alpha \sigma \beta} \nabla_{\mu}{ }^{\sigma \tau} \hat{\varphi}_{\tau}{ }^{\alpha \beta} \\
& -\frac{42}{5} \nabla_{\sigma} R_{\nu \alpha \rho \beta} \nabla_{\mu}^{\sigma \tau} \hat{\varphi}_{\tau}^{\alpha \beta}-\frac{49}{5} \nabla_{\alpha} R_{\nu \sigma \rho \beta} \nabla_{\mu}{ }^{\sigma \tau} \hat{\varphi}_{\tau}{ }^{\alpha \beta}-\frac{203}{50} \nabla^{\sigma} \hat{\varphi}^{\tau \alpha \beta} \nabla_{\mu \tau \sigma} R_{\nu \alpha \rho \beta} \\
& -\frac{21}{10} \nabla^{\sigma} \hat{\varphi}^{\tau \alpha \beta} \nabla_{\mu \tau \alpha} R_{\nu \sigma \rho \beta}-\frac{42}{25} \nabla^{\sigma} \hat{\varphi}_{\sigma}^{\tau \alpha} \nabla_{\mu}^{\beta}{ }_{\beta} R_{\nu \tau \rho \alpha}+\frac{98}{25} \nabla^{\sigma} \hat{\varphi}_{\mu}{ }^{\tau \alpha} \nabla_{\nu}{ }_{\beta} R_{\rho \tau \sigma \alpha}
\end{aligned}
$$




$$
\begin{aligned}
& -\frac{112}{25} \nabla_{\mu} \hat{\varphi}^{\sigma \tau \alpha} \nabla_{\sigma}{ }_{\beta} R_{\nu \tau \rho \alpha}+\frac{77}{25} \nabla^{\sigma} \hat{\varphi}_{\mu}{ }^{\tau \alpha} \nabla_{\sigma}{ }_{\beta} R_{\nu \tau \rho \alpha}+\frac{42}{5} \nabla_{\sigma} R_{\nu \alpha \rho \beta} \nabla^{\sigma \tau}{ }_{\tau} \hat{\varphi}_{\mu}{ }^{\alpha \beta} \\
& +\frac{56}{5} \nabla_{\alpha} R_{\nu \sigma \rho \beta} \nabla^{\sigma \tau}{ }_{\tau} \hat{\varphi}_{\mu}{ }^{\alpha \beta}-\frac{98}{5} \nabla_{\alpha} R_{\rho \sigma \tau \beta} \nabla^{\sigma \tau \alpha} \hat{\varphi}_{\mu \nu}{ }^{\beta}+\frac{399}{50} \nabla_{\nu} R_{\rho \sigma \tau \beta} \nabla^{\sigma \tau \alpha} \hat{\varphi}_{\mu \alpha}{ }^{\beta} \\
& +\frac{721}{50} \nabla_{\tau} R_{\nu \sigma \rho \beta} \nabla^{\sigma \tau \alpha} \hat{\varphi}_{\mu \alpha}{ }^{\beta}-\frac{161}{50} \nabla_{\beta} R_{\nu \sigma \rho \tau} \nabla^{\sigma \tau \alpha} \hat{\varphi}_{\mu \alpha}{ }^{\beta}-7 \nabla_{\mu} R_{\nu \sigma \rho \beta} \nabla^{\sigma \tau \alpha} \hat{\varphi}_{\tau \alpha}{ }^{\beta} \\
& +\frac{154}{25} \nabla^{\sigma} \hat{\varphi}_{\mu}{ }^{\tau \alpha} \nabla_{\tau}{ }^{\beta}{ }_{\beta} R_{\nu \sigma \rho \alpha}-\frac{36}{5} \hat{\varphi}^{\sigma \tau \alpha} \nabla_{\mu \sigma}{ }_{\beta} R_{\nu \tau \rho \alpha}-\frac{42}{5} R_{\mu}{ }^{\sigma \tau \alpha} \nabla_{\nu \rho \tau}{ }^{\beta} \hat{\varphi}_{\sigma \alpha \beta} \\
& +\frac{84}{5} R_{\mu}{ }^{\sigma \tau \alpha} \nabla_{\nu \sigma \tau}{ }^{\beta} \hat{\varphi}_{\rho \alpha \beta}+\frac{56}{5} R_{\mu}{ }^{\sigma \tau \alpha} \nabla_{\nu \tau}{ }_{\beta} \hat{\varphi}_{\rho \sigma \alpha}-\frac{42}{5} R_{\mu}{ }^{\sigma}{ }_{\nu}{ }^{\tau} \nabla_{\rho \sigma}{ }^{\alpha \beta} \hat{\varphi}_{\tau \alpha \beta} \\
& -\frac{42}{5} R_{\mu}{ }^{\sigma}{ }_{\nu}{ }^{\tau} \nabla_{\rho}{ }^{\alpha \beta}{ }_{\beta} \hat{\varphi}_{\sigma \tau \alpha}-\frac{98}{5} R_{\mu}{ }^{\sigma \tau \alpha} \nabla_{\sigma \tau}{ }^{\beta}{ }_{\beta} \hat{\varphi}_{\nu \rho \alpha}+\frac{56}{5} R_{\mu}{ }^{\sigma} \nu_{\nu}{ }^{\tau} \nabla_{\sigma}{ }^{\alpha \beta}{ }_{\beta} \hat{\varphi}_{\rho \tau \alpha} \\
& +\frac{21}{5} R_{\mu}{ }^{\sigma}{ }_{\nu}{ }^{\tau} \nabla^{\alpha}{ }_{\alpha}{ }^{\beta}{ }_{\beta} \hat{\varphi}_{\rho \sigma \tau}-\frac{2}{5} \nabla_{\mu \nu \rho}{ }^{\sigma \tau \alpha} \hat{\varphi}_{\sigma \tau \alpha}+\frac{12}{5} \nabla_{\mu \nu}{ }^{\sigma \tau \alpha}{ }_{\alpha} \hat{\varphi}_{\rho \sigma \tau} \\
& -3 \nabla_{\mu}{ }^{\sigma \tau} \tau^{\alpha}{ }_{\alpha} \hat{\varphi}_{\nu \rho \sigma}+\nabla^{\sigma}{ }_{\sigma}{ }^{\tau}{ }^{\alpha}{ }_{\alpha} \hat{\varphi}_{\mu \nu \rho}+\mathcal{O}\left(R^{2}\right),
\end{aligned}
$$

where $\nabla_{\mu_{1} \cdots \mu_{n}}=\nabla_{\left(\mu_{1}\right.} \cdots \nabla_{\left.\mu_{n}\right)}$ and $\hat{\varphi}_{\mu \nu \rho}=\varphi_{\mu \nu \rho}-\frac{1}{2} g_{(\mu \nu} \varphi_{\rho) \sigma}{ }^{\sigma}$.

\section{Conclusions}

In this paper we have studied conformal wave operators for HS fields on general backgrounds. We have found a manifestly factorized form for them in (A)dS, and for spin 2 on arbitrary Einstein backgrounds. The whole analysis has been carried out in arbitrary dimensions. The main result of this paper is the explicit form of the wave operator on (A)dS backgrounds, together with the identification of the obstruction to factorization on more general backgrounds.

The results of this paper confirm previous conjecture about conformal HS wave operators on (A)dS backgrounds [48, 62]. On the other hand the identification of the obstruction to factorization for spin $s>2$ HS wave operators on more general backgrounds lead us to reconsider modifications of this conjecture. Specifically, the computation of the $c$-coefficient of the Weyl anomaly done in [62], which assumes factorization on Ricci-flat backgrounds, should be reconsidered.

We expect the variant of the Tractor formalism exploited in this paper to be a key tool for further analysis of conformal HS theories on generic backgrounds. We plan to come back to these issues in future publications. The full form of the conformal wave operator on generic backgrounds is still missing, and so far we have been able to fix it only up to linear order in the Riemann tensor for spin 3.

Before concluding let us mention once again that the operator gauge invariance condition turns to be very powerful to control conformal invariance in any dimension. Therefore, we conjecture the existence of a solution to the latter stronger operator condition on general backgrounds. This feature can be also interpreted by saying that operator gauge invariance of the corresponding wave operator is equivalent to its conformal invariance. Since in our setting we only require linear Weyl symmetry on top of gauge symmetry, this observation shares possible similarities with analogous statements in the context of CFT (see e.g. $[72-74])$. 
It will also be interesting to address questions about interactions and gauge algebra deformations with the variant of the tractor calculus introduced here. We leave this as well as other interesting questions related to conformal HS fields for future research.

\section{Note added}

During the final stages of preparation of the present article the paper [75] by R. Metsaev appeared. Although using different techniques, it contains some results that are in overlap with the results presented in section 3.2. While we use an explicitly higher derivative formalism, [75] exploits an ordinary derivative formulation by introducing auxiliary fields. The results of [75] are equivalent to the factorization of the conformal operator in (A)dS background that we recover in a different way.

\section{Acknowledgments}

We are indebted to E. Skvortsov and S. Theisen for useful discussions and comments, and for having brought this problem to our attention. We are also grateful to R. Manvelyan and A. Sagnotti for useful discussions and comments on the manuscript. We performed various computations with the $x$ Act collection of Mathematica packages [76], and in particular with $x$ Tras [77].

\section{A Notation and conventions}

In this appendix we give a brief introduction to the techniques and conventions we used to deal with conformal HS fields.

We mainly rely on an operator formalism where index contraction and symmetrization of indices are realized in terms of auxiliary variables. This allows us to translate tensor operations in terms of operator calculus, resulting in simplified manipulations (see e.g. [78] for further details).

After replacing symmetric tensors by polynomials in the auxiliary variable $u^{a}$ as in equation (2.2), it is possible to define the action of the covariant derivative as a differential operator on both $x$ and $u$ :

$$
\begin{aligned}
\tilde{\nabla}_{\mu} \rightarrow \nabla_{\mu} & =\tilde{\nabla}_{\mu}-\frac{1}{2} \omega_{\mu b}^{a} L_{a}^{b}=\tilde{\nabla}_{\mu}-\omega_{\mu b}^{a} u^{b} \partial_{u^{a}}, \\
{\left[\nabla_{\mu}, \nabla_{\nu}\right] } & =\Lambda\left(u_{\mu} \partial_{u_{\nu}}-u_{\nu} \partial_{u_{\mu}}\right)+R_{\mu \nu \rho \sigma}^{\Lambda}(x) u^{\rho} \partial_{u_{\sigma}},
\end{aligned}
$$

where above and henceforth commutator equations will be assumed to hold on scalar functions of $u$ with no naked tensorial index. Here $\tilde{\nabla}_{\mu}$ is the standard covariant derivative acting on naked tensorial indices, $\omega$ is the spin-connection and $L_{a}^{b}$ are the Lorentz generators. We have expressed the latter in terms of differential operators upon introducing the derivative $\partial_{u^{a}}$, which is defined by:

$$
\partial_{u^{a}} u^{b}=\delta_{a}^{b} . \quad L_{b}^{a}=u^{a} \partial_{u^{b}}-u^{b} \partial_{u^{a}} .
$$


We have also expressed the commutator of covariant derivatives in terms of $R_{\mu \nu \rho \sigma}^{\Lambda}$. This is simply the Riemann tensor minus its constant trace part:

$$
R_{\mu \nu \rho \sigma}^{\Lambda}=R_{\mu \nu \rho \sigma}-\Lambda\left(g_{\mu \rho} g_{\nu \sigma}-g_{\nu \rho} g_{\mu \sigma}\right)
$$

This conveniently parametrizes the difference between constant curvature metrics and more general ones.

In what follows we shall work only with the contracted auxiliary variable $u^{\mu}=e_{a}^{\mu}(x) u^{a}$ and the associated derivative $\partial_{u^{\mu}}=e^{a}{ }_{\mu}(x) \partial_{u^{a}}$. The latter commutes with the covariant derivative on generic backgrounds as a consequence of the vielbein postulate:

$$
\left[\nabla_{\mu}, u^{\nu}\right]=0, \quad\left[\partial_{u^{\mu}}, \nabla_{\nu}\right]=0 .
$$

The operators box, symmetrized gradient, divergence, trace, symmetrized metric, and spin can then be represented respectively by the following operators:

$$
\begin{aligned}
& \text { box: } \square, \quad \text { divergence: } \nabla \cdot \partial_{u}, \quad \text { sym. metric: } u^{2} \text {, } \\
& \text { sym. gradient: } u \cdot \nabla, \quad \text { trace: } \partial_{u}^{2}, \quad \text { spin: } u \cdot \partial_{u} \text {. }
\end{aligned}
$$

They satisfy the following operator algebra:

$$
\begin{aligned}
{[\square, u \cdot \nabla]=} & \Lambda\left[u \cdot \nabla\left(2 u \cdot \partial_{u}+d-1\right)-2 u^{2} \nabla \cdot \partial_{u}\right] \\
& +2 R_{\mu \nu \rho \sigma}^{\Lambda} \nabla^{\mu} u^{\nu} u^{\rho} \partial_{u_{\sigma}}-\left(\nabla_{\sigma} R_{\nu \rho}^{\Lambda}-\nabla_{\rho} R_{\nu \sigma}^{\Lambda}\right) u^{\nu} u^{\rho} \partial_{u^{\sigma}}+R_{\nu \rho}^{\Lambda} u^{\nu} \nabla^{\rho} \\
{\left[\nabla \cdot \partial_{u}, \square\right]=\Lambda } & {\left[\left(2 u \cdot \partial_{u}+d-1\right) \nabla \cdot \partial_{u}-2 u \cdot \nabla \partial_{u}^{2}\right] } \\
& -2 R_{\mu \nu \rho \sigma}^{\Lambda} \nabla^{\mu} u^{\rho} \partial_{u^{\sigma}} \partial_{u^{\nu}}+R_{\mu \nu}^{\Lambda} \nabla^{\mu} \partial_{u^{\sigma}}+\left(\nabla^{\mu} R_{\mu \sigma}^{\Lambda}\right) \partial_{u_{\sigma}} \\
& -\left(\nabla_{\sigma} R_{\nu \rho}^{\Lambda}-\nabla_{\rho} R_{\nu \sigma}^{\Lambda}\right) u^{\rho} \partial_{u_{\sigma}} \partial_{u_{\nu}} \\
{\left[\nabla \cdot \partial_{u}, u \cdot \nabla\right]=} & +\Lambda\left[u \cdot \partial_{u}\left(u \cdot \partial_{u}+d-2\right)-u^{2} \partial_{u}^{2}\right] \\
& +R_{\mu \nu \rho \sigma}^{\Lambda} u^{\nu} u^{\rho} \partial_{u_{\mu}} \partial_{u_{\sigma}}+R_{\mu \nu}^{\Lambda} u^{\mu} \partial_{u_{\nu}} \\
{\left[\nabla \cdot \partial_{u}, u^{2}\right]=} & 2 u \cdot \nabla \\
{\left[\partial_{u}^{2}, u \cdot \nabla\right]=} & 2 \nabla \cdot \partial_{u} \\
{\left[\partial_{u}^{2}, u^{2}\right]=} & 2\left(d+2 u \cdot \partial_{u}\right) .
\end{aligned}
$$

On Einstein backgrounds these commutation relations simplify due to the identity $R_{\mu \nu \rho \sigma}^{\Lambda}=$ $W_{\mu \nu \rho \sigma}$, where $W_{\mu \nu \rho \sigma}$ is the Weyl tensor. The main difficulty is however the fact that the operator algebra does not close and requires the inclusion of Riemann tensors and their derivatives of arbitrary order. The algebra closes only if one restricts it to its spin $s$ sector.

In the case of conformal higher spin fields one needs to work with fields defined on equivalence classes,

$$
\varphi_{\mu_{1} \cdots \mu_{s}} \sim \varphi_{\mu_{1} \cdots \mu_{s}}+g_{\left(\mu_{1} \mu_{2}\right.} \alpha_{\left.\mu_{3} \cdots \mu_{s}\right)},
$$

or in terms of the auxiliary variables:

$$
\varphi \sim \varphi+u^{2} \alpha .
$$


In order to work on such equivalence classes it is quite useful to exploit a variant of the Tractor calculus (see e.g. [64] and references therein) in which one replaces ordinary derivative operators $\partial_{u}$ with Thomas-D derivatives:

$$
\hat{\partial}_{u^{\mu}}=\partial_{u^{\mu}}-\frac{1}{h} u_{\mu} \partial_{u}^{2}
$$

Here we have defined $h$ as

$$
h=d-2+2 u \cdot \partial_{u} .
$$

Thomas-D derivatives have the useful property to be automatically defined on the above equivalence classes, since

$$
\hat{\partial}_{u^{\mu}} u^{2}=u^{2}\left(\partial_{u^{\mu}}-\frac{1}{h-4} u_{\mu} \partial_{u}^{2}\right) \sim 0
$$

In this way the operator algebra simplifies since we can consistently set $u^{2} \sim 0$, and we end up with only four operators: $\square, u \cdot \nabla, \nabla \cdot \hat{\partial}_{u}$, and $u \cdot \hat{\partial}_{u}$. Notice that

$$
\hat{\partial}_{u}^{2}=u^{2}\left(\partial_{u}^{2}\right)^{2} \sim 0
$$

Further using the commutation relation

$$
\left[\hat{\partial}_{u^{\mu}}, u^{\nu}\right]=g_{\mu \nu}-\frac{2}{h} u_{\mu} \hat{\partial}_{u^{\mu}}
$$

we end up with the following operator algebra:

$$
\begin{aligned}
{\left[\nabla_{\mu}, \nabla_{\nu}\right]=} & \Lambda\left(u_{\mu} \hat{\partial}_{u_{\nu}}-u_{\nu} \hat{\partial}_{u_{\mu}}\right)+R_{\mu \nu \rho \sigma}^{\Lambda}(x) u^{\rho} \hat{\partial}_{u_{\sigma}}, \\
{[\square, u \cdot \nabla]=} & \Lambda u \cdot \nabla\left(2 u \cdot \hat{\partial}_{u}+d-1\right) \\
& -2 R_{\mu \nu \rho \sigma}^{\Lambda} u^{\mu} \nabla^{\nu} u^{\rho} \hat{\partial}_{u_{\sigma}}-u^{\nu} u^{\rho}\left(\nabla \cdot \hat{\partial}_{u} R_{\nu \rho}^{\Lambda}\right)+\left(u \cdot \nabla R_{\nu \sigma}^{\Lambda}\right) u^{\nu} \hat{\partial}_{u^{\sigma}}+R_{\mu \nu}^{\Lambda} u^{\mu} \nabla^{\nu}, \\
{\left[\nabla \cdot \hat{\partial}_{u}, \square\right]=} & \Lambda\left(2 u \cdot \hat{\partial}_{u}+d-1\right) \nabla \cdot \hat{\partial}_{u} \\
& -2 R_{\mu \nu \rho \sigma}^{\Lambda} \nabla^{\mu} u^{\rho} \hat{\partial}_{u^{\nu}} \hat{\partial}_{u^{\sigma}}+R_{\mu \nu}^{\Lambda} \nabla^{\mu} \hat{\partial}_{u^{\nu}}+\left(\nabla^{\mu} R_{\mu \sigma}^{\Lambda}\right) \hat{\partial}_{u_{\sigma}} \\
& +u^{\rho} \hat{\partial}_{u_{\nu}}\left(\nabla \cdot \hat{\partial}_{u} R_{\nu \rho}^{\Lambda}\right)-\left(u \cdot \nabla R_{\nu \sigma}^{\Lambda}\right) \hat{\partial}_{u_{\nu}} \hat{\partial}_{u_{\sigma}}, \\
{\left[\nabla \cdot \hat{\partial}_{u}, u \cdot \nabla\right]=} & \square-\frac{2}{h} u \cdot \nabla \nabla \cdot \hat{\partial}_{u}+\Lambda u \cdot \hat{\partial}_{u}\left(u \cdot \hat{\partial}_{u}+d-2\right) \\
& -R_{\mu \nu \rho \sigma}^{\Lambda} u^{\mu} u^{\rho} \hat{\partial}_{u_{\nu}} \hat{\partial}_{u_{\sigma}}+R_{\mu \nu}^{\Lambda} u^{\mu} \hat{\partial}_{u_{\nu}} .
\end{aligned}
$$

This operator algebra is defined on equivalence classes (A.8), and again closes only if one also includes derivatives of the Riemann tensor and their commutators recursively.

\section{B Spin s wave operator in standard tensor notation}

It is not too difficult to present the generic recursive structure of the two derivative operators entering the (A)dS solution in terms of standard tensor notation. One can then define 
the following recursion relation

$$
\begin{aligned}
\varphi_{\mu(s)}^{(i-1)}= & \mathcal{P}_{\mu(s)}{ }^{\nu(s)}\left\{\left[\square-\Lambda[(i-s+1)(i-s-d+2)-s] \varphi_{\nu(s)}^{(i)}\right.\right. \\
& \left.-\frac{d-4+2 s}{i(d-3-i+2 s)}\left[s \nabla_{\nu} \nabla^{\alpha} \varphi_{\alpha \nu(s-1)}^{(i)}+\frac{s(s-1)}{d-4+2 s} \nabla_{\nu} \nabla_{\nu} \varphi_{\alpha \nu(s-2)}^{(i) \alpha}\right]\right\},
\end{aligned}
$$

where eliminating the auxiliary variable acting with the operator $\left(\hat{\partial}_{u_{\mu}}\right)^{s}$, we are left with the spin s traceless projector $\mathcal{P}_{\mu(s)}{ }^{\nu(s)}$. Above, we have conveniently defined new fields $\varphi_{\mu(s)}^{(i-1)}$ with $\varphi_{\mu(s)}^{(0)}=\mathcal{O}_{\mu(s)}^{(s)}$ and $\varphi_{\mu(s)}^{\left(\frac{d}{2}-2+s\right)}=\varphi_{\mu(s)}$ of weight shifting by two units at each step. One then ends up with the conformal operator written in standard tensor notation upon substituting the corresponding fields above till expressing $\varphi_{\mu(s)}^{(0)}$ in terms of $\varphi_{\mu(s)}$.

\section{Wave operator in non factorized form}

In this appendix we will rewrite the factorized wave operator for a conformal spin $s$ field on (A)dS backgrounds in a more standard form from which one can read off the analogue of the de Donder tensor for conformal higher spins.

We start by writing an Ansatz of the type:

$$
\begin{aligned}
\mathcal{O}^{(s)} & =\sum_{i=0}^{s+\frac{d}{2}-2} \gamma_{i}(u \cdot \nabla)^{i} \mathcal{B}_{s+\frac{d}{2}-2-i}\left(\nabla \cdot \hat{\partial}_{u}\right)^{i} \\
& =\sum_{i=0}^{s+\frac{d}{2}-2} \gamma_{i}(u \cdot \nabla)^{i}\left[\prod_{j=1}^{s+\frac{d}{2}-2-i}\left(\square+\beta_{i, j} \Lambda\right)\right]\left(\nabla \cdot \hat{\partial}_{u}\right)^{i} .
\end{aligned}
$$

A useful trick is then to parameterize the gauge variation of a divergence as:

$$
\left(\nabla \cdot \hat{\partial}_{u}\right)^{n} u \cdot \nabla=\left[a_{n} \square+b_{n} u \cdot \nabla \nabla \cdot \hat{\partial}_{u}+\Lambda c_{n}\right]\left(\nabla \cdot \hat{\partial}_{u}\right)^{n-1},
$$

where the coefficients satisfy the following recursion relations

$$
\begin{aligned}
& a_{n}=a_{n-1}+b_{n-1}, \\
& b_{n}=b_{n-1}\left(1-\frac{2}{d-2+2(s-n)}\right), \\
& c_{n}=c_{n-1}+b_{n-1}(s-n)(s-n+d-2)+a_{n-1}(2(s-n)+d-1),
\end{aligned}
$$

with

$$
a_{1}=1, \quad b_{1}=-\frac{2}{d-4+2 s}, \quad c_{1}=(s-1)(s+d-3),
$$

and hence

$$
\begin{aligned}
& a_{n}=1-\frac{n(n-1)}{d-4+2 s}+\frac{(n-1)(n-2)}{d-6+2 s}, \\
& b_{n}=-\frac{\frac{d}{2}-2+s-n}{\left(\frac{d}{2}-2+s\right)\left(\frac{d}{2}-3+s\right)},
\end{aligned}
$$


while we do not present the solution for $c_{n}$ for brevity. One can now compute the gauge variation of the operator $\mathcal{E}_{i}=\gamma_{i}(u \cdot \nabla)^{i} \mathcal{B}_{s+\frac{d}{2}-2-i}\left(\nabla \cdot \hat{\partial}_{u}\right)^{i}$ :

$$
\begin{aligned}
\mathcal{E}_{i} u \cdot \nabla= & \gamma_{i} a_{i}(u \cdot \nabla)^{i}\left[\prod_{j=1}^{s+\frac{d}{2}-2-i}\left(\square+\Lambda \beta_{i, j}\right)\right]\left(\square+\Lambda \frac{c_{i}}{a_{i}}\right)\left(\nabla \cdot \hat{\partial}_{u}\right)^{i-1} \\
& +\gamma_{i} b_{i}(u \cdot \nabla)^{i+1}\left[\prod_{j=1}^{s+\frac{d}{2}-2-i}\left[\square+\Lambda\left(\beta_{i, j}+2(s-i)+d-3\right)\right]\right]\left(\nabla \cdot \hat{\partial}_{u}\right)^{i} .
\end{aligned}
$$

Therefore, by requiring that the terms proportional to $(u \cdot \nabla)^{i+1}$ in the variation of $\mathcal{E}_{i}$ cancel the terms proportional to $(u \cdot \nabla)^{i+1}$ in the variation of $\mathcal{E}_{i+1}$ one gets the following conditions for the free coefficients $\gamma_{i}$ and $\beta_{i, j}$ :

$$
\begin{aligned}
\gamma_{i+1} & =-\frac{b_{i}}{a_{i+1}} \gamma_{i}, \\
\beta_{i, 1} & =\frac{c_{i+1}}{a_{i+1}}-2(s-i)-d+3, \\
\beta_{i, j} & =\beta_{i+1, j-1}-2(s-i)-d+3 .
\end{aligned}
$$

The conditions can be solved to give

$$
\begin{aligned}
\gamma_{i} & =(-1)^{i} \frac{\prod_{n=0}^{i-1} b_{n}}{\prod_{n=1}^{i} a_{n}}, \quad \gamma_{0}=1, \\
\beta_{i, 1} & =\frac{c_{i+1}}{a_{i+1}}-2(s-i)-d+3, \\
\beta_{i, j} & =\beta_{i+j-1,1}-(j-1)[2(s-i)+d-j-1] .
\end{aligned}
$$

After plugging in the solution for the coefficients $a_{i}, b_{i}$ and $c_{i}$ we then get

$$
\beta_{i, j}=(i+j+1-s)(i+j-s-d+2)-(j-1)[2(s-i)+d-j-1]-s .
$$

As before, this matches all partially massless points in $d=4$, and also some massive points in higher dimensions.

The generalized de Donder tensor can be easily extracted from equation (C.1):

$$
\mathcal{D}_{\left(\frac{d}{2}-3+s\right)}=\sum_{i=1}^{\frac{d}{2}-2+s}(u \cdot \nabla)^{i-1} \mathcal{B}_{s+\frac{d}{2}-2-i}\left(\nabla \cdot \hat{\partial}_{u}\right)^{i} \varphi_{(s)} .
$$

This tensor has one derivative less than the full equation of motion. From the gauge invariance condition one can easily extract its gauge variation:

$$
\delta_{\xi} \mathcal{D}_{\left(\frac{d}{2}-3+s\right)}=-\prod_{j=1}^{\frac{d}{2}-2+s}\left(\square+\left(\beta_{0, j}+2 s+d-3\right) \Lambda\right) \varepsilon_{(s-1)} .
$$

The right-hand-side can be viewed as a second order equation on an effective gauge parameter that is of order $2 s+d-6$. This linear second order diagonal equation can be solved throughout spacetime [79] in order to set $\mathcal{D}_{\left(\frac{d}{2}-3+s\right)}$ to zero. In this partial gauge, the equation of motion becomes (3.19). 


\section{Examples in various dimensions}

In this appendix we list some known non-linear conformal actions, and confirm that their equations of motion reduce to (2.4) upon linearization on (A)dS spaces.

\section{D.1 Spin 1 data}

$\boldsymbol{d}=\mathbf{2}$. The 2 dimensional case is trivial since the spin 1 conformal field does not propagate and indeed the number of derivatives compatible with conformal symmetry is 0 .

$\boldsymbol{d}=4$. In four dimensions the Maxwell's theory is conformally invariant, and its equation of motion is precisely (C.1) for $s=1$ and $d=4$.

$\boldsymbol{d}=\mathbf{6}$. In six dimensions there are a number conformal invariants quadratic in $A=\varphi_{(1)}$. Yet there is only one that is gauge invariant, not a total derivative, and non-zero on AdS backgrounds. It reads

$$
I=F^{\mu \nu}\left(\left(\square-\frac{1}{2} R\right) \delta_{\mu}^{\rho} \delta_{\nu}^{\sigma}+R_{\mu}^{\rho} \delta_{\nu}^{\sigma}+C_{\mu \nu}^{\rho \sigma}\right) F_{\rho \sigma}+\nabla_{\mu} J^{\mu},
$$

with $F_{\mu \nu}=\nabla_{[\mu} A_{\nu]}$. The Weyl tensor could have been omitted, as $F \cdot C \cdot F$ is conformally invariant on its own. However, including it reproduces Branson's $D_{4,1}$ conformal operator [51] acting on $A_{\mu}$ as the equation of motion:

$$
\nabla^{\nu}\left(\nabla_{[\mu} \nabla^{\rho} F_{\nu] \rho}+S F_{\mu \nu}-4 S_{[\mu}^{\rho} F_{\nu] \rho}\right)=0,
$$

where $S_{\mu \nu}$ is the Schouten tensor and $S$ is its trace. Upon linearizing these equations of motion on (A)dS we find (2.4) or (C.1) for $s=1$ and $d=6$ in agreement with the solution to the operator gauge invariance condition.

\section{D.2 Spin 2 data}

$\boldsymbol{d}=\mathbf{2}$. Two-dimensional conformal gravity is just Einstein gravity, whose linearized equation of motion on (A)dS can be precisely recast in the form (2.4) or (C.1) for $s=2$ and $d=2$.

$\boldsymbol{d}=4$. The action for four dimensional conformal gravity is

$$
S=\int d^{4} x \sqrt{-g} C_{\mu \nu \rho \sigma} C^{\mu \nu \rho \sigma}
$$

whose linearized equation of motion is exactly (2.4) or (C.1) for $s=2$ and $d=4$.

$\boldsymbol{d}=\mathbf{6}$. In six dimensions there are three conformal invariants for gravity, namely [68, 70, $71]$

$$
\begin{aligned}
& I_{1}=C_{\mu \rho \sigma \nu} C^{\mu \alpha \beta \nu} C_{\alpha}{ }^{\rho \sigma}{ }_{\beta}, \\
& I_{2}=C_{\mu \nu \rho \sigma} C^{\rho \sigma \alpha \beta} C_{\alpha \beta}{ }^{\mu \nu}, \\
& I_{3}=C_{\mu \rho \sigma \lambda}\left(\delta_{\nu}^{\mu} \square+4 R^{\mu}{ }_{\nu}-\frac{6}{5} \delta_{\nu}^{\mu} R\right) C^{\nu \rho \sigma \lambda}+\nabla_{\mu} J^{\mu}
\end{aligned}
$$


with $\nabla_{\mu} J^{\mu}$ a total derivative which can be found in [68]. Because the Weyl tensor vanishes on AdS backgrounds, only the third invariant gives a non-zero quadratic perturbation on AdS. Upon computing its equations of motion, we find (2.4) or (C.1) for $s=2$ and $d=6$, again in agreement with the general result obtained above enforcing the stronger operator gauge invariance condition.

Open Access. This article is distributed under the terms of the Creative Commons Attribution License (CC-BY 4.0), which permits any use, distribution and reproduction in any medium, provided the original author(s) and source are credited.

\section{References}

[1] M. Kaku, P.K. Townsend and P. van Nieuwenhuizen, Properties of conformal supergravity, Phys. Rev. D 17 (1978) 3179 [inSPIRE].

[2] E. Bergshoeff, M. de Roo and B. de Wit, Extended conformal supergravity, Nucl. Phys. B 182 (1981) 173 [inSPIRE].

[3] E.S. Fradkin and A.A. Tseytlin, One loop $\beta$-function in conformal supergravities, Nucl. Phys. B 203 (1982) 157 [inSPIRE].

[4] E.S. Fradkin and A.A. Tseytlin, Asymptotic freedom in extended conformal supergravities, Phys. Lett. B 110 (1982) 117 [INSPIRE].

[5] S. Deser and R.I. Nepomechie, Gauge invariance versus masslessness in de Sitter space, Annals Phys. 154 (1984) 396 [INSPIRE].

[6] S. Deser and R.I. Nepomechie, Anomalous propagation of gauge fields in conformally flat spaces, Phys. Lett. B 132 (1983) 321 [inSPIRE].

[7] R.J. Riegert, The particle content of linearized conformal gravity, Phys. Lett. A 105 (1984) 110 [inSPIRE].

[8] E.S. Fradkin and A.A. Tseytlin, Conformal supergravity, Phys. Rept. 119 (1985) 233 [INSPIRE].

[9] N. Boulanger and M. Henneaux, A derivation of Weyl gravity, Annalen Phys. 10 (2001) 935 [hep-th/0106065] [INSPIRE].

[10] N. Boulanger, M. Henneaux and P. van Nieuwenhuizen, Conformal (super)gravities with several gravitons, JHEP 01 (2002) 035 [hep-th/0201023] [INSPIRE].

[11] N. Boulanger, A Weyl-covariant tensor calculus, J. Math. Phys. 46 (2005) 053508 [hep-th/0412314] [INSPIRE].

[12] E.S. Fradkin and V.Y. Linetsky, Superconformal higher spin theory in the cubic approximation, Nucl. Phys. B 350 (1991) 274 [INSPIRE].

[13] A.Y. Segal, Conformal higher spin theory, Nucl. Phys. B 664 (2003) 59 [hep-th/0207212] [INSPIRE].

[14] O.V. Shaynkman, I.Y. Tipunin and M.A. Vasiliev, Unfolded form of conformal equations in $M$ dimensions and $o(M+2)$ modules, Rev. Math. Phys. 18 (2006) 823 [hep-th/0401086] [INSPIRE].

[15] R.R. Metsaev, Ordinary-derivative formulation of conformal totally symmetric arbitrary spin bosonic fields, JHEP 06 (2012) 062 [arXiv:0709.4392] [INSPIRE]. 
[16] M.A. Vasiliev, On conformal, $\mathrm{SL}(4, \mathbb{R})$ and $\mathrm{Sp}(8, R)$ symmetries of $4 d$ massless fields, Nucl. Phys. B 793 (2008) 469 [arXiv:0707.1085] [INSPIRE].

[17] M.A. Vasiliev, Bosonic conformal higher-spin fields of any symmetry, Nucl. Phys. B 829 (2010) 176 [arXiv:0909.5226] [INSPIRE].

[18] I. Florakis, D. Sorokin and M. Tsulaia, Higher spins in hyperspace, arXiv:1401.1645 [INSPIRE].

[19] B. de Wit and D.Z. Freedman, Systematics of higher spin gauge fields, Phys. Rev. D 21 (1980) 358 [INSPIRE].

[20] M.A. Vasiliev, Higher spin gauge theories: star product and AdS space, hep-th/9910096 [INSPIRE].

[21] X. Bekaert, S. Cnockaert, C. Iazeolla and M.A. Vasiliev, Nonlinear higher spin theories in various dimensions, hep-th/0503128 [INSPIRE].

[22] X. Bekaert, N. Boulanger and P. Sundell, How higher-spin gravity surpasses the spin two barrier: no-go theorems versus yes-go examples, Rev. Mod. Phys. 84 (2012) 987 [arXiv: 1007.0435] [INSPIRE].

[23] A. Sagnotti, Notes on strings and higher spins, J. Phys. A 46 (2013) 214006 [arXiv: 1112.4285] [INSPIRE].

[24] V.E. Didenko and E.D. Skvortsov, Elements of Vasiliev theory, arXiv:1401.2975 [INSPIRE].

[25] R.G. Leigh and A.C. Petkou, $\mathrm{SL}(2, \mathbb{Z})$ action on three-dimensional CFTs and holography, JHEP 12 (2003) 020 [hep-th/0309177] [INSPIRE].

[26] R.R. Metsaev, Shadows, currents and AdS, Phys. Rev. D 78 (2008) 106010 [arXiv: 0805.3472] [INSPIRE].

[27] R.R. Metsaev, Gauge invariant two-point vertices of shadow fields, AdS/CFT and conformal fields, Phys. Rev. D 81 (2010) 106002 [arXiv:0907.4678] [INSPIRE].

[28] X. Bekaert, E. Joung and J. Mourad, Effective action in a higher-spin background, JHEP 02 (2011) 048 [arXiv: 1012.2103] [INSPIRE].

[29] E. Joung and J. Mourad, Boundary action of free AdS higher-spin gauge fields and the holographic correspondence, JHEP 06 (2012) 161 [arXiv:1112.5620] [INSPIRE].

[30] A.R. Gover and A. Waldron, Boundary calculus for conformally compact manifolds, arXiv: 1104.2991 [INSPIRE].

[31] M.A. Vasiliev, Holography, unfolding and higher-spin theory, J. Phys. A 46 (2013) 214013 [arXiv: 1203.5554] [INSPIRE].

[32] S. Giombi, I.R. Klebanov, S.S. Pufu, B.R. Safdi and G. Tarnopolsky, AdS description of induced higher-spin gauge theory, JHEP 10 (2013) 016 [arXiv:1306.5242] [INSPIRE].

[33] E. Joung and M. Taronna, Cubic-interaction-induced deformations of higher-spin symmetries, JHEP 03 (2014) 103 [arXiv:1311.0242] [INSPIRE].

[34] R.R. Metsaev, Ordinary-derivative formulation of conformal low spin fields, JHEP 01 (2012) 064 [arXiv:0707.4437] [InSPIRE].

[35] J. Maldacena, Einstein gravity from conformal gravity, arXiv:1105.5632 [INSPIRE].

[36] H. Lü, Y. Pang and C.N. Pope, Conformal gravity and extensions of critical gravity, Phys. Rev. D 84 (2011) 064001 [arXiv:1106.4657] [INSPIRE]. 
[37] C. Fronsdal, Massless fields with integer spin, Phys. Rev. D 18 (1978) 3624 [InSPIRE].

[38] V.E. Lopatin and M.A. Vasiliev, Free massless bosonic fields of arbitrary spin in d-dimensional de Sitter space, Mod. Phys. Lett. A 3 (1988) 257 [INSPIRE].

[39] A. Pashnev and M. Tsulaia, Description of the higher massless irreducible integer spins in the BRST approach, Mod. Phys. Lett. A 13 (1998) 1853 [hep-th/9803207] [INSPIRE].

[40] I.L. Buchbinder, A. Pashnev and M. Tsulaia, Lagrangian formulation of the massless higher integer spin fields in the AdS background, Phys. Lett. B 523 (2001) 338 [hep-th/0109067] [INSPIRE].

[41] A. Sagnotti and M. Tsulaia, On higher spins and the tensionless limit of string theory, Nucl. Phys. B 682 (2004) 83 [hep-th/0311257] [INSPIRE].

[42] D. Francia and A. Sagnotti, Minimal local Lagrangians for higher-spin geometry, Phys. Lett. B 624 (2005) 93 [hep-th/0507144] [INSPIRE].

[43] E.D. Skvortsov and M.A. Vasiliev, Geometric formulation for partially massless fields, Nucl. Phys. B 756 (2006) 117 [hep-th/0601095] [INSPIRE].

[44] I.L. Buchbinder, A.V. Galajinsky and V.A. Krykhtin, Quartet unconstrained formulation for massless higher spin fields, Nucl. Phys. B 779 (2007) 155 [hep-th/0702161] [INSPIRE].

[45] E.D. Skvortsov and M.A. Vasiliev, Transverse invariant higher spin fields, Phys. Lett. B 664 (2008) 301 [hep-th/0701278] [INSPIRE].

[46] D. Francia, J. Mourad and A. Sagnotti, (A)dS exchanges and partially-massless higher spins, Nucl. Phys. B 804 (2008) 383 [arXiv:0803.3832] [INSPIRE].

[47] A. Campoleoni and D. Francia, Maxwell-like lagrangians for higher spins, JHEP 03 (2013) 168 [arXiv: 1206.5877] [INSPIRE].

[48] E. Joung and K. Mkrtchyan, A note on higher-derivative actions for free higher-spin fields, JHEP 11 (2012) 153 [arXiv:1209.4864] [INSPIRE].

[49] J. Erdmenger, Conformally covariant differential operators: properties and applications, Class. Quant. Grav. 14 (1997) 2061 [hep-th/9704108] [INSPIRE].

[50] J. Erdmenger and H. Osborn, Conformally covariant differential operators: symmetric tensor fields, Class. Quant. Grav. 15 (1998) 273 [gr-qc/9708040] [INSPIRE].

[51] T.P Branson, Differential operators canonically associated to a conformal structure, Math. Scand. 57 (1985) 293.

[52] C.R. Graham, Conformal powers of the laplacian via stereographic projection SIGMA 3 (2007) 121 [arXiv: 0711.4798].

[53] C.R. Graham et al., Conformally invariant powers of the Laplacian. I. Existence, J. London Math. Soc. 46 (1992) 557.

[54] A.R. Gover, Laplacian operators and Q-curvature on conformally Einstein manifolds, Math. Ann. 336 (2006) 311.

[55] A. Higuchi, Forbidden mass range for spin-2 field theory in de Sitter space-time, Nucl. Phys. B 282 (1987) 397 [InSPIRE].

[56] A. Higuchi, Symmetric tensor spherical harmonics on the $N$ sphere and their application to the de Sitter group $\mathrm{SO}(N, 1)$, J. Math. Phys. 28 (1987) 1553 [Erratum ibid. 43 (2002) 6385]. [INSPIRE]. 
[57] S. Deser and A. Waldron, Partial masslessness of higher spins in (A)dS, Nucl. Phys. B 607 (2001) 577 [hep-th/0103198] [InSPIRE].

[58] S. Deser and A. Waldron, Null propagation of partially massless higher spins in $(A) d S$ and cosmological constant speculations, Phys. Lett. B 513 (2001) 137 [hep-th/0105181] [INSPIRE].

[59] K. Alkalaev and M. Grigoriev, Unified BRST approach to (partially) massless and massive AdS fields of arbitrary symmetry type, Nucl. Phys. B 853 (2011) 663 [arXiv:1105.6111] [INSPIRE].

[60] S. Deser, E. Joung and A. Waldron, Partial masslessness and conformal gravity, J. Phys. A 46 (2013) 214019 [arXiv:1208.1307] [inSPIRE].

[61] X. Bekaert and M. Grigoriev, Higher order singletons, partially massless fields and their boundary values in the ambient approach, Nucl. Phys. B 876 (2013) 667 [arXiv:1305.0162] [INSPIRE].

[62] A.A. Tseytlin, On partition function and Weyl anomaly of conformal higher spin fields, Nucl. Phys. B 877 (2013) 598 [arXiv:1309.0785] [INSPIRE].

[63] C. Aragone and S. Deser, Consistency problems of hypergravity, Phys. Lett. B 86 (1979) 161 [INSPIRE].

[64] E. Joung, M. Taronna and A. Waldron, A calculus for higher spin interactions, JHEP 07 (2013) 186 [arXiv:1305.5809] [INSPIRE].

[65] E.S. Fradkin and M.A. Vasiliev, On the gravitational interaction of massless higher spin fields, Phys. Lett. B 189 (1987) 89 [InSPIRE].

[66] N. Boulanger, S. Leclercq and P. Sundell, On the uniqueness of minimal coupling in higher-spin gauge theory, JHEP 08 (2008) 056 [arXiv:0805.2764] [INSPIRE].

[67] O.A. Gelfond and M.A. Vasiliev, Operator algebra of free conformal currents via twistors, Nucl. Phys. B 876 (2013) 871 [arXiv:1301.3123] [INSPIRE].

[68] F. Bastianelli, G. Cuoghi and L. Nocetti, Consistency conditions and trace anomalies in six-dimensions, Class. Quant. Grav. 18 (2001) 793 [hep-th/0007222] [INSPIRE].

[69] N. Boulanger and J. Erdmenger, A classification of local Weyl invariants in $D=8$, Class. Quant. Grav. 21 (2004) 4305 [hep-th/0405228] [INSPIRE].

[70] R.R. Metsaev, 6d conformal gravity, J. Phys. A 44 (2011) 175402 [arXiv:1012.2079] [INSPIRE].

[71] J. Oliva and S. Ray, Classification of six derivative lagrangians of gravity and static spherically symmetric solutions, Phys. Rev. D 82 (2010) 124030 [arXiv:1004.0737] [INSPIRE].

[72] A. Dymarsky, Z. Komargodski, A. Schwimmer and S. Theisen, On scale and conformal invariance in four dimensions, arXiv:1309.2921 [INSPIRE].

[73] A. Bzowski and K. Skenderis, Comments on scale and conformal invariance in four dimensions, arXiv: 1402.3208 [INSPIRE].

[74] A. Dymarsky, K. Farnsworth, Z. Komargodski, M.A. Luty and V. Prilepina, Scale invariance, conformality and generalized free fields, arXiv:1402.6322 [INSPIRE].

[75] R.R. Metsaev, Arbitrary spin conformal fields in (A)dS, arXiv:1404.3712 [INSPIRE]. 
[76] J.M. Martín-García et al., xAct: efficient tensor computer algebra for Mathematica, http://xact.es/.

[77] T. Nutma, xTras: a field-theory inspired xAct package for mathematica, Comput. Phys. Commun. 185 (2014) 1719 [arXiv:1308.3493] [INSPIRE].

[78] M. Taronna, Higher-spin interactions: three-point functions and beyond, arXiv:1209.5755 [INSPIRE].

[79] S.W. Hawking and G.F.R. Ellis, The large scale structure of space-time, Cambridge University Press, Cambridge U.K. (1973). 